\title{
High performance fibres and the mechanical attributes of cut resistant structures made therewith
}

\author{
S. Rebouillat \& B. Steffenino \\ DuPont International S.A., Geneva, P.O. Box 50, Geneva, Switzerland
}

\begin{abstract}
High performance materials are rarely used as $100 \%$ compound, therefore most of the time when reference is made to these categories of materials it is as a matter of fact a reference to a system comprising a well-combined ingredients exhibiting together high performances in use. Fibres, micro-fibres and now nanofibres are definitely part of the high performance reinforcing ingredients, which in a system largely contributes to the performance in use of the designed system.

High performance fibres, and more specifically aramids for their outstanding heat and mechanical properties, although most often used as reinforcing compound, are also used more uniquely and directly in situations where mechanical and thermal protections are required. It is therefore useful as a preliminary grounding to review these types of materials in terms of their properties. Then their attributes and potential contribution alone or in a system become more specific and can be extrapolated more easily. The mechanical performance and more specifically the cut resistance of articles made of high performance materials are generally measured against several norm specifications. Those are not designed to predict the "real" in use performance of the considered protective articles. This gap is even larger when the cut resistance of the compounding fibre increases. Although relationships exist between the various cut performance levels obtained with the ASTM, ISO and EN norms (respectively American, International and European standards), there is a need for a more fundamental understanding and interpretation of the data. The ASTM and ISO approaches remain the most suitable methods for that purpose. Various parameters, such as the high performance material and fibre composition, the effect of kinetic such as the cutting speed and the speed profile, the force distributions, the artefacts generated by various surface coatings are presented and discussed aiming at improving the interpretations of the data generated through the norm procedures. Therewith, significant progress in the understanding of the cut fundamentals and the norm harmonization are made available to the users of high performance fibres, such as KEVLAR ${ }^{\circledR}$.

Keywords: high performance materials and fibres, textile structures, coated textile materials, mechanical protection, cut mechanism, cut fundamentals, KEVLAR®.
\end{abstract}




\section{Introduction}

Aromatic polyamides came as breakthrough materials in commercial applications as early as 1961, with the market launch of the meta-aramid fibre Nomex ${ }^{\circledR}$, which opened new horizons in the field of thermal and electrical insulation.

A much higher tenacity and modulus fibre was developed and commercialised, also by DuPont, under the trade name KEVLAR ${ }^{\circledR}$ in 1971. Scientists, in the fields of liquid crystals, polymers, rheology and fibre processing, as well as, process and system engineers spent several years prior and during the early stage of its market introduction to establish the basics and fundamental understanding necessary to take full advantage of this new class of high performance materials. The outstanding potential of these materials derived mostly from the anisotropy of their superimposed substructures presenting pleated, crystalline, fibrillar and skin-core characteristics.

In this work we will focus on the main high performance fibres and the applications of the commercial aramids. Basic papers outlining the engineering of such products, especially their surface modifications, are available and provide a good basis for a scientific background [1-7].

We will study in more detail the protective solutions obtained from high performance fibres. A gentle force of $0.01 \mathrm{~N}$ applied on a razor blade, such as the one used by the hairdresser, represents more than 1000 bar or $10^{4}\left(\mathrm{~N} / \mathrm{cm}^{2}\right)$, there is no doubt that the performance of an effective cut protective barrier has to be a high performance.

In the professional activities the awareness and the prevention regarding hand injuries have substantially contributed to contain the injury level, nonetheless the hand protection remains an area where the room for improvement is substantial. Thanks to the personal protective equipment, such as gloves, made of high performance fibres, the situation is improving on a worldwide basis. General guidance about hand protection is provided in the ANSI/ISEA 105-2000 [8].

Norms, developed in 1987 in Europe and in 1997 in the USA, have played an important role in classifying cut resistant materials. Glove performance can now reach the upper range of the cut scale level defined by certain norms with a substantial dexterity enhancement.

Norm levels are nowadays considered more as entry criteria in the proposition of a given hand protection than as a truly predictive tool to anticipate the real cut risk where experience and expert judgment prevail.

The fundamental understanding of the cut mechanism also plays an essential role to complement this approach. The test equipment, which are described in the various norms relative to cut protection, such as ASTM, EN and ISO (respectively American, European and International standards), are good tools to develop a basis for the cut fundamental understanding. The work presented in this paper provides and illustrates a number of situations where cut fundamentals can be link with the properties of high performance fibres.

There is no attempt to extrapolate the results to a real risk encountered in the field, therefore this study cannot constitute a basis for risk analysis but can serve 
as guidance towards an improved understanding of factors which may influence the cut performance of protective equipment.

\section{High performance fibres}

\subsection{Main high performance fibres}

The following designation was adopted in 1974 by the United States Federal Trade Commission to describe the aromatic polyamide based fibres under the generic term aramid: "a manufactured fibre in which the fibre forming substance is a long chain synthetic polyamide in which at least $85 \%$ of the amide (- CO - NH -) linkages are attached directly to two aromatic rings".

The earliest representative of this class is poly-m-phenyleneisophthalamide, which was commercialised by DuPont in 1967 as Nomex ${ }^{\circledR}$ aramid fibre. Its chemical formula is:<smiles>CN1CCN(C)C2(CC1)COC2c1cccc(C(=O)Nc2cccc(N)c2)c1</smiles>

The discovery in 1965 of high tenacity, high modulus fibres from liquid crystalline solutions of synthetic para-aromatic polyamides led to the commercial production of KEVLAR ${ }^{\circledR}$ aramid fibre by DuPont Co in 1971[9]; the corresponding chemical formula is given below:

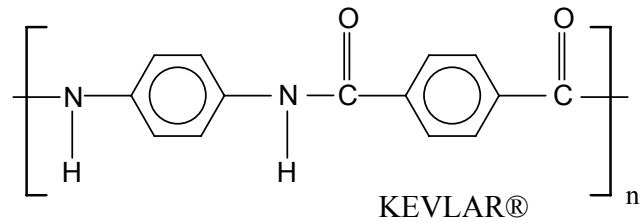

KEVLAR $®$ fibres are poly (p-phenylene terephthalamide) (PPTA) [10].

Another para-aramid, Twaron ${ }^{\circledR}$, similar to KEVLAR $\AA$, and an aromatic copolyamide appeared on the market towards the end of the 80s. Teijin commercialised the copolyamide Technora ${ }^{\circledR}$ fibre.

For the sake of completeness, Monsanto in the 1970s developed, based on an aromatic polyamide-hydrazine composition, an aromatic copolyamide fibre under the code X500 which almost reached the market.

PBO (commercialised under the trade name Zylon ${ }^{\circledR}$ ) and PIPD, often referred as to M5, are other high performance fibres sufficiently close to the aramids to be mentioned here [1].

\subsection{Spinning fibres}

Production of fibres initially involves heating the spinning polymeric solution up to a suitable processing temperature, which is of the order of $80^{\circ} \mathrm{C}$ for the highly 
concentrated solutions in 100\% (water-free) [11] sulphuric acid. The solution state corresponds to a nematic liquid crystalline phase. The concentration limit for the polymer in spinning solution is $20 \mathrm{wt} \%$.

Polymer spinning solutions are extruded through spinning holes and subjected to elongational stretch across a small air gap, illustrated in fig. 1. The spinning holes fulfill an important function. Under shear, the crystal domains become elongated and orientated in the direction of the deformation [12-14]. Once in the air gap, elongational stretching takes place. This is effected by making the velocity of the fibre as it leaves the coagulating bath higher than the velocity of the polymer as it emerges from the spinning holes. This ratio is often referred to as the draw ratio, which can be fine-tuned to obtain higher tenacities and moduli with lower elongations and denier. The resulting stretch in the air gap further perfects the respective alignment of the liquid crystal domains.

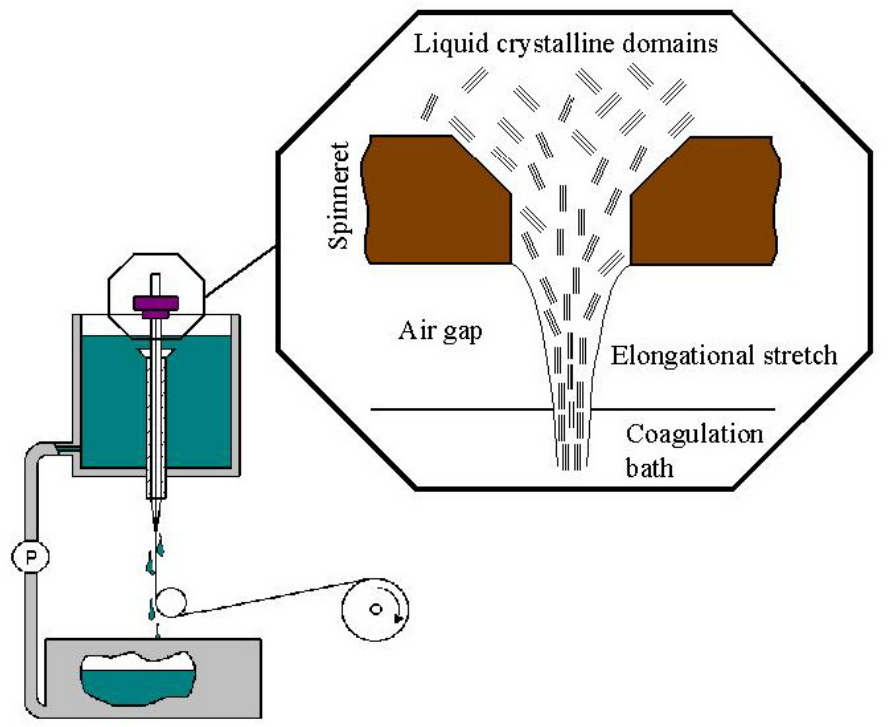

Figure 1: Schematical representation of the extrusion of the liquid crystalline solution in the dry-jet wet spinning process.

Overall a higher polymer orientation in the coagulation medium corresponds to higher mechanical properties of the fibre. Because of the slower relaxation time of these liquid crystal systems, the high as-spun fibre orientation can be attained and retained via coagulation with cold water [15]. Essentially, the crystallinity and orientation of the solution are translated to the fibre. These factors allow the production of high strength, high modulus, as spun fibres.

\subsection{Structures and properties}

Aramid fibres have unique properties that set them apart from other fibres. Aramid fibre tensile strength and modulus are significantly higher than earlier 
organic fibres and fibre elongation is lower. Aramid fibres can be woven on fabric looms more easily than brittle fibres such as glass, carbon or ceramic. They also exhibit inherent resistance to organic solvents, fuels, lubricants and exposure to flame.

The superimposed structures, such as the crystallites, the fibrils and the skincore boundaries, are definitely unique attributes, which can be partially tailored through the fibre process engineering.

\subsection{Mechanical properties}

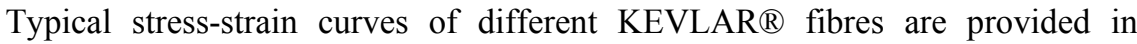
fig. 2(a), which clearly outlines the modulus increase from KEVLAR ${ }^{\circledR} 29$ to KEVLAR $₫ 149$. There are differences between KEVLAR $₫ 49$ and KEVLAR $\AA$ 29 . The respective moduli, brought by various spinning conditions and posttreatments performed on para-aramid precursors are generally considered as intermediate between those of graphite, boron and glass fibres. The linear stressstrain behaviour of para-aramids is special compared to most man-made fibres which tensile behaviours are depicted in fig. 2(b).

(a)

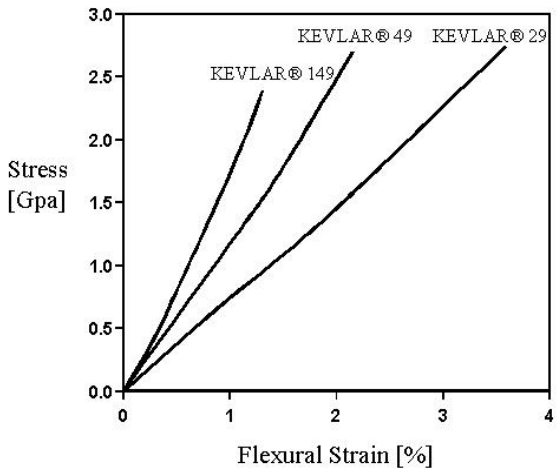

(b)

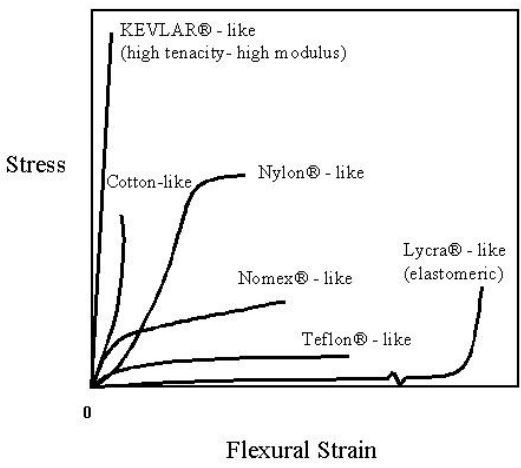

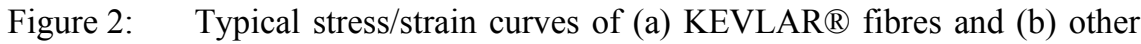
commercially representative industrial yarns.

Creep is measured either by the length variation under tension or by the stress decrease at constant gage length. Para-aramids, which exhibit little creep, differ significantly from other highly oriented polymeric fibres, such as HMPE fibres, which can break after several days under intermediate load due to their high creep properties associated with a stress slip of molecules compared to a structure tightening in the case of para-aramids. The temperature, the load relative to the fibre ultimate strength, the water content and other parameters affect creep. 


\subsection{Applications}

The present section takes into consideration a selected range of applications of the aramids with an attempt to underline some of the system engineering aspects. This approach may provide not only a better understanding of the major reasons for these fibres to contribute to the best balanced performance of the system, but also may stimulate new ideas in the way these fibres are used in the considered system or how they could be used in a new one.

As an example, the understanding of how a para-aramid fibre can be shaped into an optimised fabric pattern to resist to a fragment impact, can provoke new ideas regarding the engineering of these fibres to offer cut and heat protection in other sectors where the kinetics may be much closer to static conditions.

This system approach, led to the development of some of the aramid fibre applications to be outlined in this section illustrated in table 1 [11].

Table 1: $\quad$ Aramid market segments and key attributes.

\begin{tabular}{|c|c|c|}
\hline Enduse & End-use systems & Key attributes \\
\hline \multirow[t]{6}{*}{ Composites } & F abrics for aircraft \& containers & Light weight \\
\hline & Pressure vessels & High strength \\
\hline & Ship building & High m odulus \\
\hline & Sport goods & Good im pact strength \\
\hline & Plastics additive & Wear resistance \\
\hline & Civil engineering & \\
\hline \multirow[t]{5}{*}{ Protective Apparels } & Heat resistance workwear & Heat resistance \\
\hline & Fire blankets & Flame retardation \\
\hline & Flame retardant textiles & Cut resistance \\
\hline & Cut protective gloves & \\
\hline & Cut protective seat covers layers & \\
\hline \multirow[t]{6}{*}{ Tires } & Truck and Aircraft tires & Low density \\
\hline & High speed tires & Weight saving \\
\hline & Motorcycle tires & High tenacity \\
\hline & and bicycle tires & Dimensional stability \\
\hline & & Low shrinkage \\
\hline & & Puncture resistance \\
\hline \multirow{5}{*}{$\begin{array}{l}\text { Mechanical Rubber Goods } \\
\text { (MRG) }\end{array}$} & Conveyor belts & High strength \\
\hline & Transmission belts & High m odulus \\
\hline & Hoses for automotive & Dimensional stability \\
\hline & Hydraulic hoses & Thermal resistance \\
\hline & $\begin{array}{l}\text { Hoses in off-shore } \\
\text { Umbilicals }\end{array}$ & Chemical resistance \\
\hline \multirow[t]{4}{*}{ Friction Products and $\mathrm{G}$ askets } & Brake linings & Fiber fibrillation \\
\hline & $\begin{array}{l}\text { Clutch racings } \\
\text { Gaskets }\end{array}$ & Chemical resistance \\
\hline & Thix otr opic additive & Low inflamm ability \\
\hline & Industrial paper & Mechanical performance \\
\hline \multirow[t]{6}{*}{ Ropes and Cables } & Aerial optical fiber cable & High strength \\
\hline & Traditional optical fiber cable & High m odulus \\
\hline & E1ectrocable & Dimensional stability \\
\hline & Mechanical construction cable & Low density \\
\hline & Mooring ropes & Corrosion resistance \\
\hline & & $\begin{array}{l}\text { Good dielectric properties } \\
\text { Heat resistance }\end{array}$ \\
\hline \multirow[t]{5}{*}{ Life Protection } & Bullet proof vests & High tenacity \\
\hline & Helm ets & High energy dissipation \\
\hline & Property protection panels & Low density and weight reduction \\
\hline & V ehicle protection & Comfort \\
\hline & Strategic equipm ent shielding & \\
\hline
\end{tabular}

The associated fig. 3 provides a concrete illustration of the dynamic network established since the inception of the aramids. This network outlines the dynamic 
integration of aramids in advanced and modern technologies with a constant adaptation to the new challenges associated with various generations of product.

There are end-use market segments, which have been quite rethought because of the aramids, such as the asbestos replacement by para-aramid pulps. There are other areas, which will continue to evolve because of the ever-growing stringent requirements for energy saving in transportation for example.

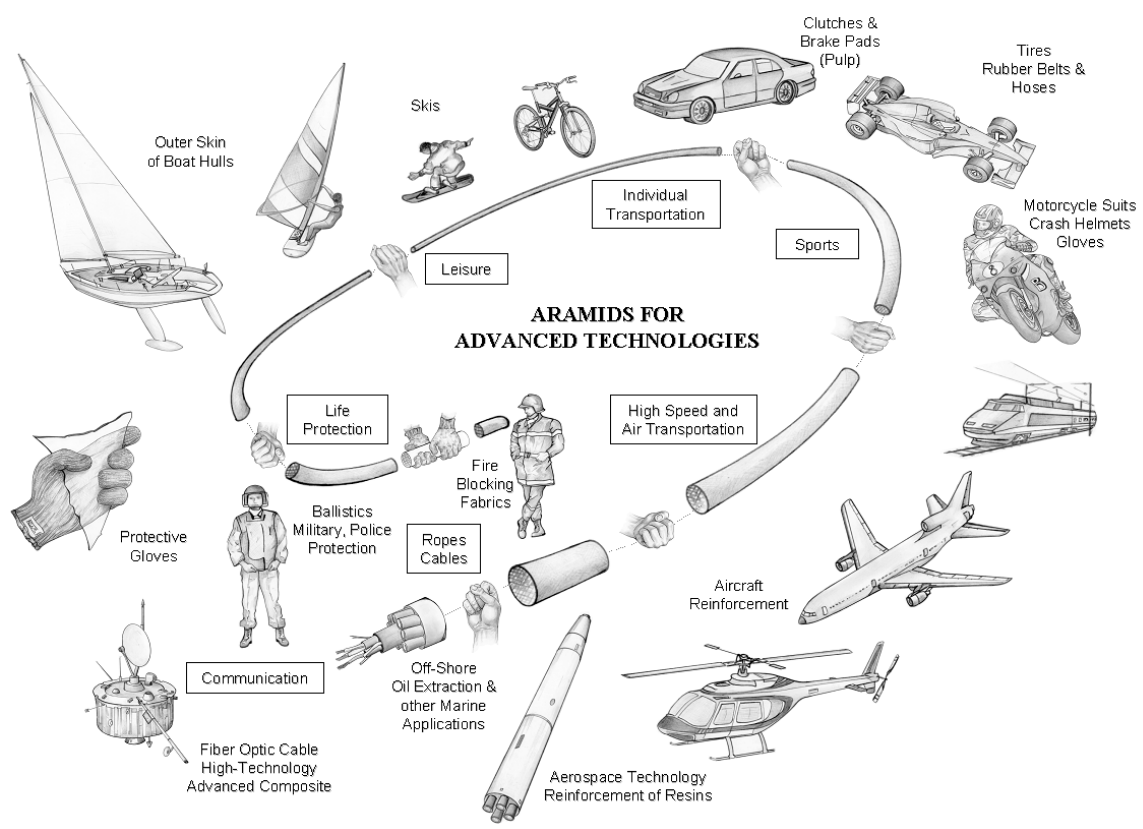

Figure 3: Applications of para-aramid fibres.

The aramid contribution in this sector is outstanding. Clearly, communications, including transportation as well as transmission, leisure and sports, life protection and, health and safety in general, have been tremendously improved and adapted to modern technologies in part because of the aramids.

\section{High performance fibres for protective solutions}

The cut performance of articles made of high performance materials is generally measured against several norm specifications $[16,17]$. Those are not designed to predict the "real" in use performance of the considered protective articles. This gap is even larger when the cut resistance of the compounding fibre increases.

Although relationships exist between the various cut performance levels obtained with the ASTM, ISO and EN norms, there is a need for a more fundamental understanding and interpretation of the data. The ASTM and ISO approaches remain the most suitable methods for cut level exceeding 3. 


\section{EN 388 (Europe) Couptest}
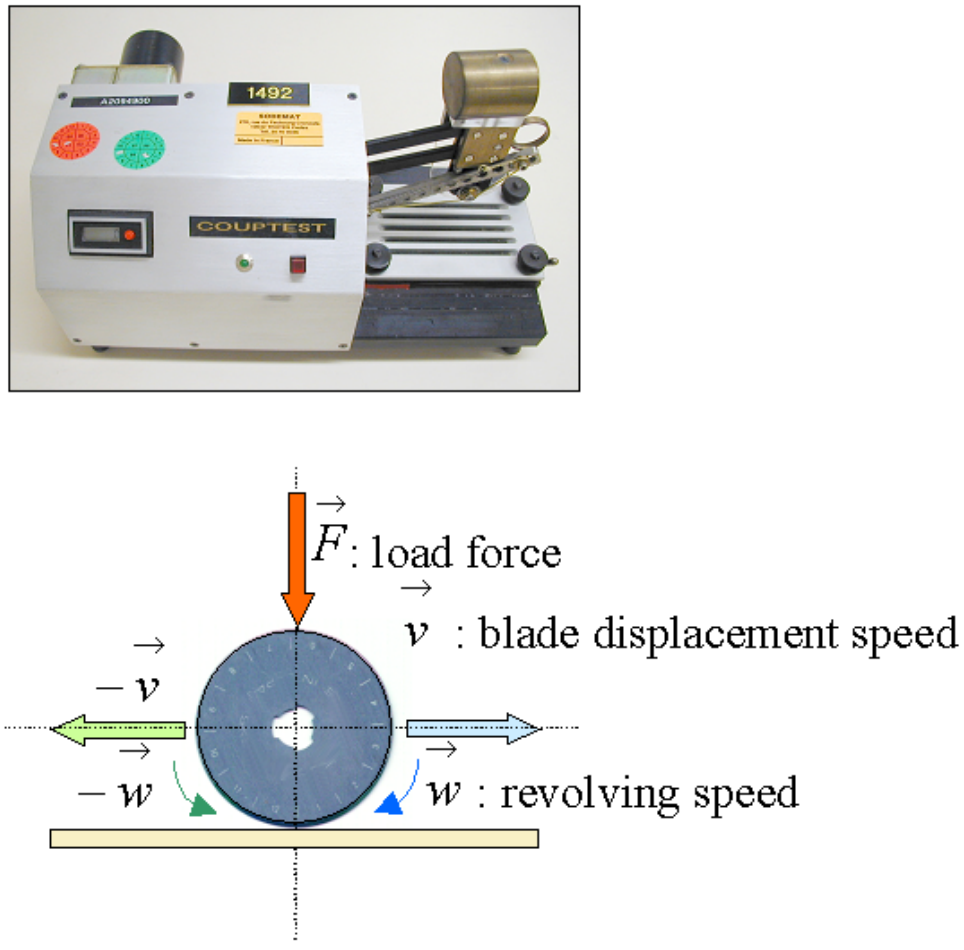

\section{Circular blade}

Figure 4(a): Test equipments (top) with schematic representation (bottom) of the force application, (EN388).

\subsection{Cut testing methods EN, ISO, ASTM}

The first standardized cut test method was developed in France in 1987 (prEN388 [18], hereafter called EN) followed by a method developed in 1997 by the American Society for Testing Material, (ASTM- F1790-97 [19], hereafter called ASTM) and its most recent international adaptation via the International 
Standardisation Organization (ISO-13997 [20], hereafter called ISO). General guidance about hand protection is summarized in the ANSI/ISEA 105-2000 [8], which relates to theses various norms.

Each testing procedure is described in the norm documents, [18-20], mentioned above. The three related test equipments are depicted in figs. 4 (a), 4(b) with a schematic representation of the blade orientation, the load force application and the blade displacement.

There are similarities between the ASTM and ISO procedures and equipments, but there are fundamentals differences between those two and the EN equipment and test method.

ASTM F1790 (USA) CPPT
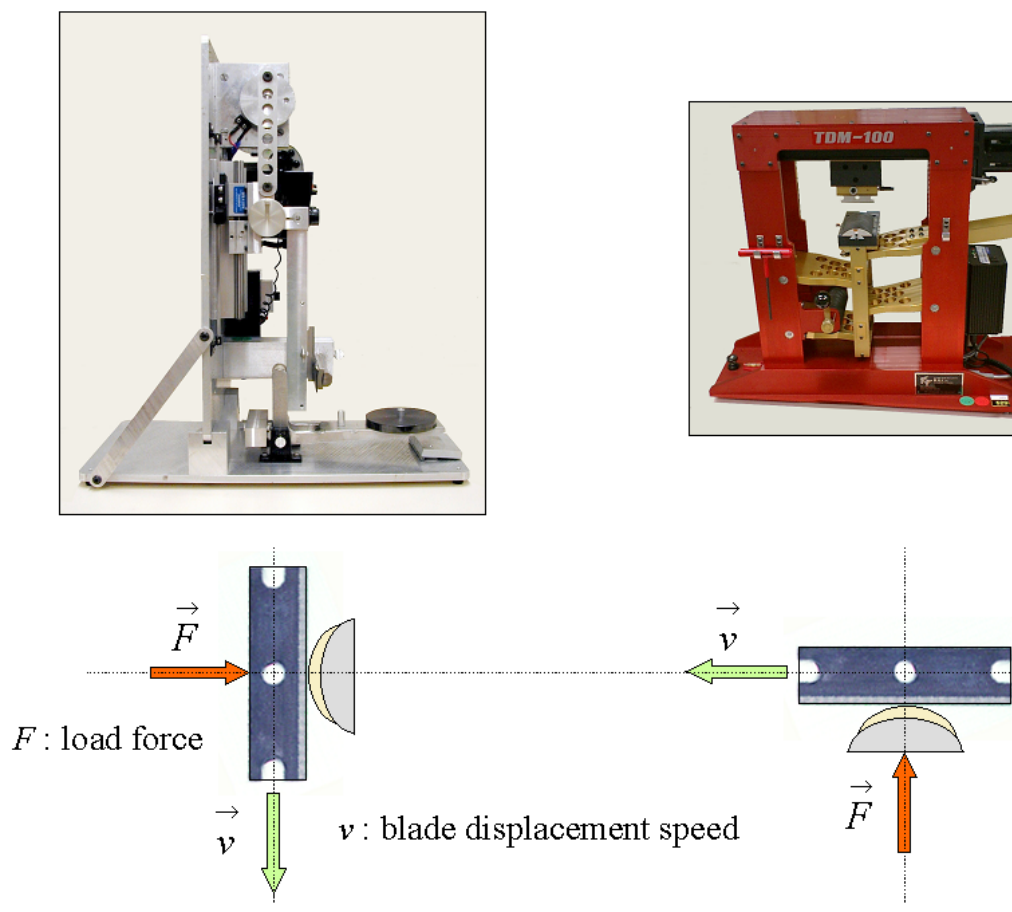

Rectangular blade
ISO 13997 (International) TDM-100

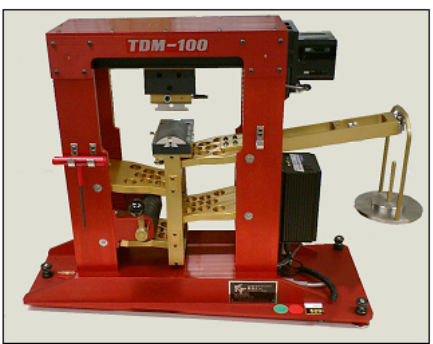

Rectangular blade

Figure 4(b): Test equipments (top) with schematic representation (bottom) of the force application, blade displacement and blade orientation, (ASTM/ ISO).

The EN test method is based on the measurement of the substrate cut resistance when submitted to the cutting effect generated by the rotation of the circular blade on the sample tested, which is under a relatively moderate pressure 
provided by a $5 \mathrm{~N}$ load force applied on the blade. The number of revolutions of the blade to cause the cut-through of the specimen will be used to calculate the cut index and therewith the cut performance. A cotton fabric is used as the reference material.

The ASTM and ISO methods on the opposite are based on the measurement of the load necessary to provoke the cut-through of the sample after a sliding distance of the rectangular blade of $20 \mathrm{~mm}$ (most recently adopted harmonized distance). The corresponding load can reach, for knitted samples made of high performance fibres, the equivalent of 1000 to $3500 \mathrm{~g}$, i.e. 10 to $35 \mathrm{~N}$, which is 2 to 7 times higher than the load force applied on the circular blade in the case of the EN method. This is a difference worth highlighting.

\subsection{Experimental part}

The design of the experiments was selected in order to clarify and better understand some of the aspects, which were partly introduced in the materials and methods section.

For the EN test method, the blade cutting edge degradation during the course of the testing of high cut performance materials, hereafter HCP materials, has been illustrated and studied by conducting tests on a "stop and go" basis, i.e. changing the blade during the test every 1,2,3, and 5 cycles as illustrated in table 2. Both $100 \%$ KEVLAR $®$ and $5.5 \%$ steel reinforced para-aramid knitted structures were used.

Table 2: EN test method: the blade cutting edge degradation profile during the course of the testing of high cut performance materials.

\begin{tabular}{ccccc}
\hline $\begin{array}{c}\text { Blade replacement } \\
\text { every (1/10 cycles) }\end{array}$ & $\begin{array}{c}\text { Cn: } \\
\text { Control specimen } \\
\text { before } \\
\text { (1/10 cycles) }\end{array}$ & $\begin{array}{c}\text { Tn: } \\
\text { Sample tested } \\
(1 / 10 \text { cycles })\end{array}$ & $\begin{array}{c}\text { Cn+1: } \\
\text { Control specimen } \\
\text { after } \\
(1 / 10 \text { cycles) }\end{array}$ & In : Cut index \\
\hline no change & 10 & 444 & 47 & 16.5 \\
30 & 9 & 235 & 44 & 9.8 \\
20 & 10 & 162 & 34 & 8.3 \\
10 & 9 & 179 & 41 & 9.1 \\
\hline
\end{tabular}

The analysis of the influence of PVC dots, melted and cured onto the knitted surface to enhance the grip properties of the fabric or the gloves, was performed using the EN and the ASTM procedures. This further illustrates some of the specific features of the two methods. Fig. 5 provides the resulting data outlining NL1, which is the load, in g, provoking the cut through of the specimen for a blade motion of $25.4 \mathrm{~mm}$.

The motion of the blade on the sample tested has been studied in terms of speed using the ASTM and ISO blade calibration material, i.e. a $1.6 \mathrm{~mm}$ neoprene layer, and 100\% para-aramid felt, which characteristics are indicated in fig. 6(a) and 6(b).

As depicted in fig. 7 a load cell, linked to a computer, was placed in the balanced arm holding the blade in order to estimate the force opposing the blade motion during the ASTM testing. Therewith the ratio of this resisting force to the applied load force has been calculated for various materials, such as neoprene, 
100\% KEVLAR ${ }^{\circledR}$ knitted gloves and another HCP material. Results are summarized in fig. 8

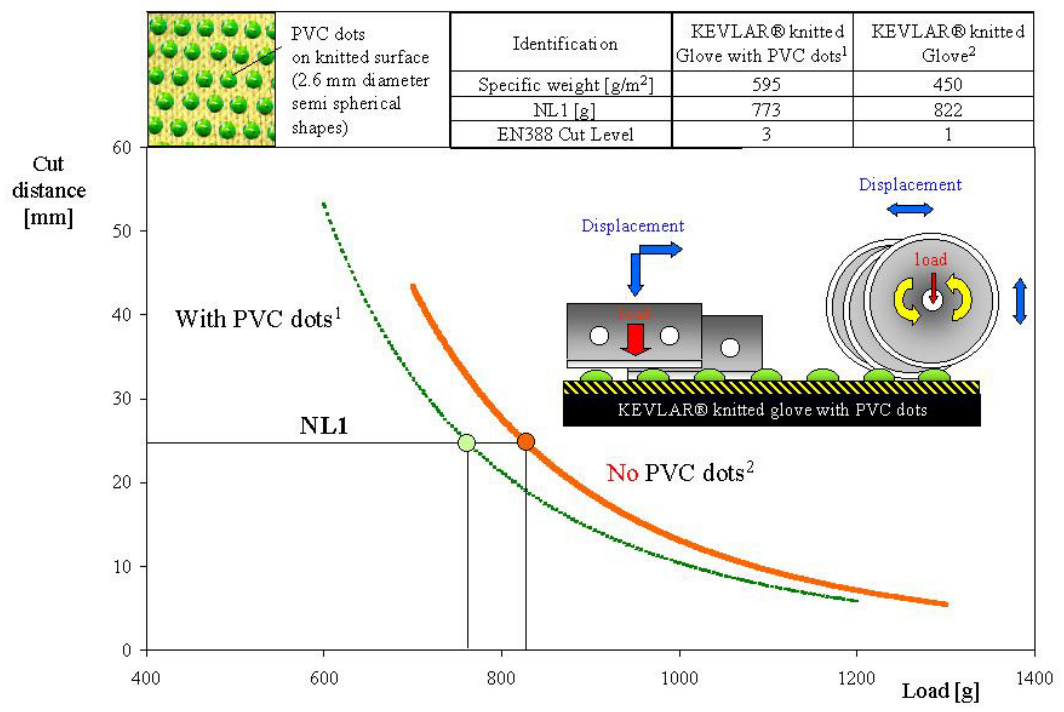

Figure 5: PVC dots influence on the EN (top table) and the ASTM results (top table and curves).

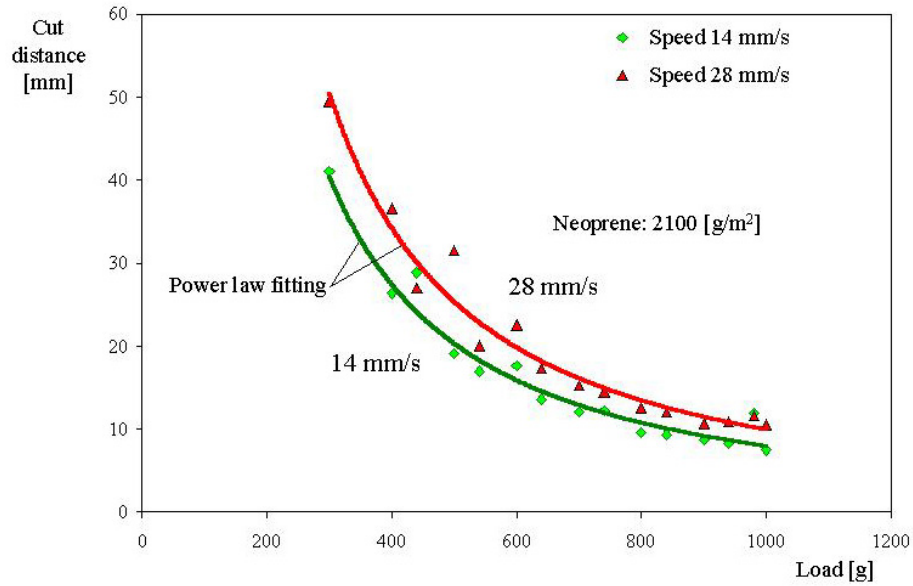

Figure 6(a): Blade speed influence on the cut performance of the calibration material. 


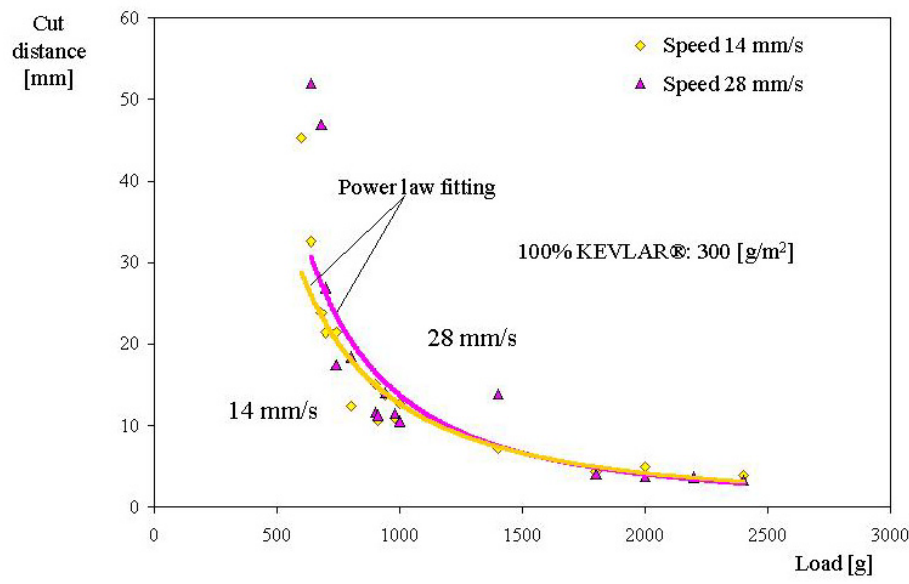

Figure 6(b): Blade speed influence on the cut performance of a $100 \%$ paraaramid textile.

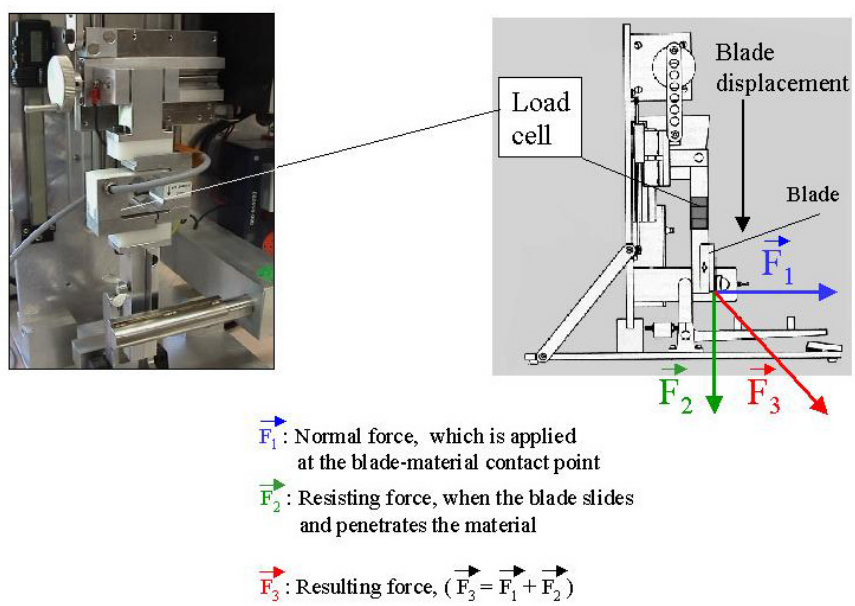

Figure 7: $\quad$ CPPT (ASTM) equipped with a compressive load cell.

Finally, a series of experiments was run to estimate the similitude between cutting and sliding process. In fig. 12 a sliding experimental assembly is depicted and was made in order to estimate the sliding force. A large part of the force applied onto a cutting device can be dissipated via the sliding friction, associated sometime with the compression and the relaxation of the materials under cut. Therefore it is important to relate the cut forces measured during cut with the 
more easily accessible sliding and friction forces. It is not our intention to pretend that a direct liaison can be established between these two physical phenomena but, in view of the lack of published data on the subject matter, it is valuable to bring another piece of comprehension to this scientific matter of relevant value to personal protection. During an accidental cut the sliding attributes of the personal protective equipment are determinant.

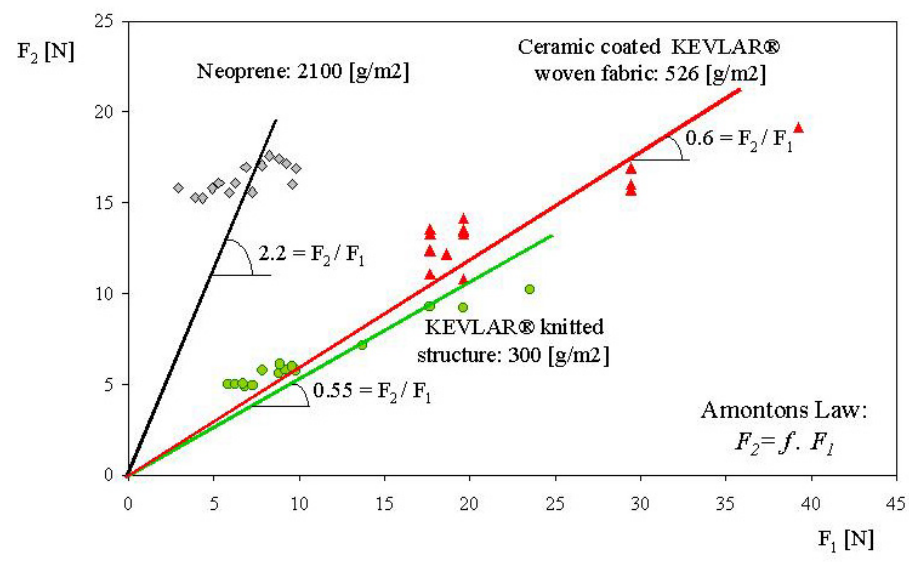

Figure 8: $\quad \mathrm{F}_{2} / \mathrm{F}_{1}$ ratio for various materials submitted to ASTM testing.

\section{Recommended for :}
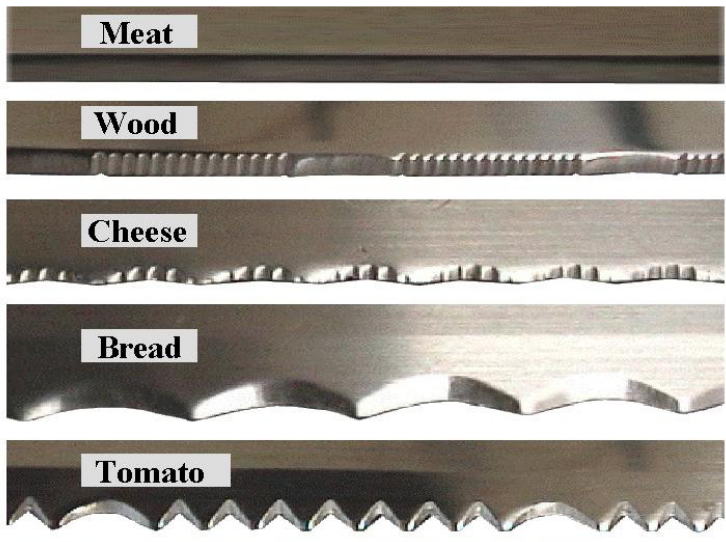

\section{All purpose}

Figure 9: Various knife cutting edge profiles and recommended uses. 


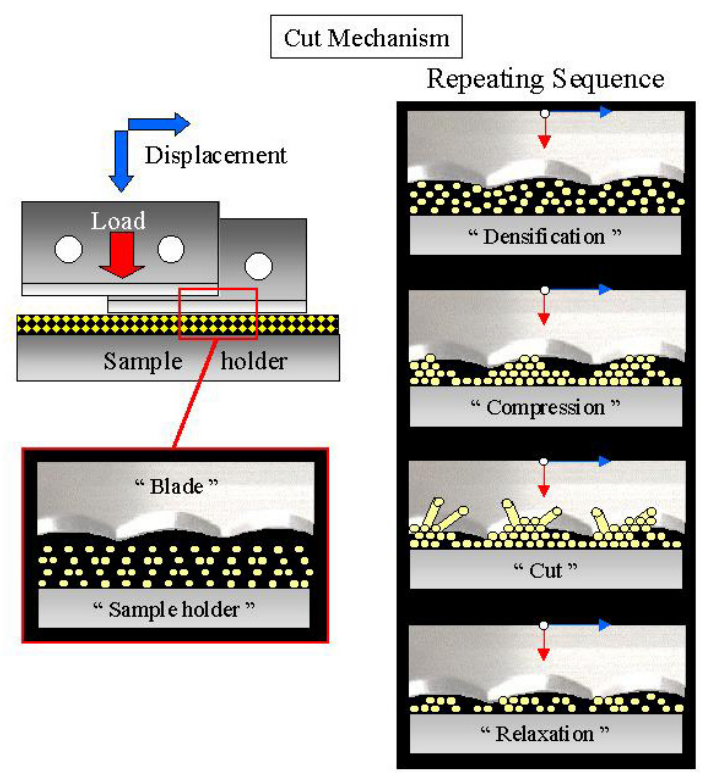

Figure 10: A proposed cut mechanism sequence: "Densification-CompressionCut-Relaxation-Densification...".

\subsection{Results and discussion}

The cut mechanism and related fundamentals are rarely described in the literature. The large diversity of situations where cuts are involved and the rather large number of physical phenomena superimposed in a cutting operation, render the scientific approach further more difficult.

There are some qualitative reflection points, which may guide the novice and help the reader through this section.

Materials, which are generally considered as cut resistant materials, are also exhibiting:

- a good tear resistance

- a reasonable puncture resistance towards sharp but not too thin perforators

- a certain resistance to abrasion or ability to fibrillate

In the cutting process itself one may consider the following as useful considerations:

the amount of material per unit volume, which the cutting threat has to go through

the deformability of this material and the tendency for this material to maintain a high density under pressure still keeping its flexibility

the mobility of the fibres on which the blade is sliding; a revolving material being more difficult to cut.

the blade dulling effect of the material being cut. Glass is a good example. 
the eventual surface or bulk phase changes occurring during the cut either due to local heating under friction or heating.

- the water or liquid retention and absorbency of the protective barrier.

Fibres with skin-core structures are generally more protective. The thickness of the skin is a positive attribute. Para-aramid fibres are cut resistant.

Rather brittle materials tend to be less effective. On the other hand ductility can be a good attribute. Steel and glass tend to support these two statements.

As exhibited in fig. 9 the design of blade profiles, leaving apart the steel selection and processing, remains an art. For example what are the criteria taken into account to make an effective knife cutting edge to cut tomatoes versus bread versus meat versus cheese, versus wood, versus textile, etc.

When one observes a few cutting edges, it seems, as depicted in fig. 10, that the sequence of -1- material blockage-densification and compression followed by -2- the effective sliding and cut of the previously compressed materials and -3the relaxation and separation of the "already cut material" is a sequence which may be taken into account to better understand the selection of a large proportion of cutting edge profiles. That densification-cut-relaxation sequence repeats itself along the blade as the cutting operation proceeds. The threshold compression ratio at which cut can progressively take place depends on the fibre mobility, the deformation/cut speed, the local humidity as well.

\subsubsection{Blade degradation during EN testing}

Using the EN norm, the degradation effect of the materials on the blade cutting edge was already demonstrated by Lara [21] with a series of test performed on fibreglass reinforced glove samples. According to this study, a fraction of cycle a cycle being represented by one blade full rotation counter-clockwise and its reverse rotation clockwise - was initially sufficient to cut the cotton calibration fabric. The same blade, after running for 50 revolution cycles on the HCP glasscontaining material, then cut the reference cotton fabric after 25 cycles compared to 0.8 cycles initially. This shows that less than a cycle was necessary to dull the blade.

Glass fibres are known for their dulling effect. A major concern shall be outlined regarding products, which have a dulling effect. Shall they be considered as effective high cut performance materials?

A partial answer is established by the fact that the ASTM/ISO norms are more and more used for these types of materials. These norms being less sensitive to the dulling effect since one spot of the blade is in contact with the sample to cut only once. The EN norm recent revisions suggest the use of the ISO testing in such cases. This is a significant step and improvement.

\subsubsection{Speed effect on cut resistance}

In which proportion the blade speed can affect one material more than another. For example figs. 6(a), 6(b) compares the speed effect, on a neoprene rubber layer and a KEVLAR ${ }^{\circledR}$ sample stamped out of a knitted glove, for a speed variation ranging from 14 to $28 \mathrm{~mm} / \mathrm{s}$.

Such a variation affects much more the neoprene load-to-blade displacement curve than the load-to-blade displacement relationship of the para-aramid 
samples. This may raise some concern about the $2.5 \mathrm{~mm} / \mathrm{s}$ versus the $110 \mathrm{~mm} / \mathrm{s}$ blade displacement speed respectively selected for the ISO and the EN procedures. The speed selected for the ASTM approach, i.e. $14 \mathrm{~mm} / \mathrm{s}$, and the highest speed of $28 \mathrm{~mm} / \mathrm{s}$ selected in the present study lies in between the ISO and the EN selected values. Should those values be reconsidered and harmonized? This question goes beyond the scope of this study.

Nonetheless, some additional data will be discussed later on and may converge with this observation providing some elements of explanation. One can already underline that in a textile structure, the fibres are relatively free to move, especially when the textile is knitted which adds bulk to the structure. This mobility can compensate for the blade displacement speed variation since the fibre elements provoke an interface slippage.

Since these standards have been put together mostly for the testing of hand protective equipment mostly made of HCP fibres such as para-aramids, the results shown in fig. 6(b) tend to support the rather limited effect of speed within the range tested. Although the emergence of dipped supported gloves may provoke some more questioning of the speed effect since a dipped or coated glove is no more than a textile structure covered by a rubber layer.

\subsubsection{Coating/printing effect on the cut resistance of high performance textile materials}

The testing of gloves with PVC dots is reported in fig. 5. For the dotted side, the level 3 given by the EN cut testing is not consistent with the $770 \mathrm{~g}$ load necessary to provoke the cut through after a $25 \mathrm{~mm}$ blade displacement using ASTM method. One would expect at least a load of 1100 g, i.e. 1.5 folds, to reach an EN level 3. Furthermore, the non-coated side of the glove, i.e. without PVC dots, reach an inferior level 1versus level 3 for the dotted side. This does not seem to make sense in terms of cut performance.

For the ASTM analysis, one observes that, for the non-dotted side, the necessary load to cut through after a $25 \mathrm{~mm}$ blade displacement is $820 \mathrm{~g}$. This is more in line with the expected results since the coating is not a cut resistant material and is a spot by spot treatment. Overall in this specific case, the EN testing largely over-estimates the real cut performance.

The EN testing does not appear to reflect the real cut performance of the material. Since it tends to over-estimate it, precautions shall be taken regarding the interpretation of the results. In terms of ASTM or ISO testing, if the polymeric layer deposited on the textile substrate is continuous and interlocked into this substrate, the cut performance is likely to be less than the substrate alone since the fibres mobility is restricted. This is not the observation made from fig. 5 since the PVC dotting is discontinuous.

The blade dulling, the speed effect and the interference of non-cut performing polymeric coating have been discussed and may find some additional explanations in the study, which follows.

\subsubsection{Forces involved during cutting/sliding process}

The apparatus designed to measure the cut performance as per the ASTM procedure is shown in fig. 4 along with a schematic diagram, which provides the 
blade orientation and displacement, and force application point. This force is generated by weights placed on a plate mounted on a lever arm assembly in a way that doubles the resulting force applied to the specimen. The test apparatus also consists of a motor-driven balanced arm, which holds the blade.

In order to measure the co-linear force component resisting to the displacement of the blade, a $50 \mathrm{~N}$ (or $200 \mathrm{~N}$ ) load cell, type S2 from HBM Germany, was installed in the arm holding the blade as per the diagram of fig. 7 . This load cell was connected to a computer interface, module MP55 from HBM Germany. The resisting force, $-\left\|\vec{F}_{2}\right\|$, was recorded during the cutting process using the CATMAN® software from HBM Germany. The normal force, $\left\|\vec{F}_{1}\right\|$, applied onto the blade edge is assumed to be directly proportional to the double weight force value, which is an approximation since during the displacement, and due to the thickness of the sample, the blade cutting edge does not remain strictly perpendicular to the sample contacting point. This is depicted in fig. 7. Fig. 8 provides various $\left\|\vec{F}_{2}\right\| /\left\|\vec{F}_{1}\right\|$ ratio.

For the neoprene calibration material the resisting force, $-\left\|\vec{F}_{2}\right\|$, is about 2.2 times superior to the normal applied force, $\left\|\vec{F}_{1}\right\|$.

The resisting force found in this study is almost equal to the one, identified as a frictional force, reported by Massé et al. [22] in his study of the basic principles used in the development of a new cut-test machine now specified in the ISO test method.

Therefore, our findings, although conducted independently on a different testing apparatus, ASTM versus ISO, are fully in line with the work conducted by Massé et al. [22] at the IRSST, Québec Canada. This is a good demonstration of the interchangeableness of the two equipments now both recommended in the ASTM norm.

Fig. 8 also provides the $\left\|\vec{F}_{2}\right\| /\left\|\vec{F}_{1}\right\|$ ratio for a KEVLAR ${ }^{\circ}$ knitted structure and a ceramic coated para-aramid woven fabric.

The two ratios are more or less $1 / 4$ of the neoprene, measured ratio which may bring a question regarding the suitability this rubber material in the ASTM and ISO norms as a blade sharpness normalizing factor.

Furthermore, the amount of friction generated to cut trough neoprene may explain why a dotted knitted structure exhibits an artificially high cut EN performance. The necessity to use the blade cutting edge only once during a test is essential in a situation where abrasion and resulting friction of the surface of the tested material are high.

The apparatus designed to measure the sliding forces is shown in fig. 11 along with a schematic diagram, very close to the fig. 7 .

In this case the resisting force is generated in part by the weight placed onto the specimen but also in part by the specimen surface characteristics inclusive of 
its deformability under pressure. The resisting force is measured during the linear displacement at a constant controlled speed equal to $4.2 \mathrm{~mm} / \mathrm{s}$.
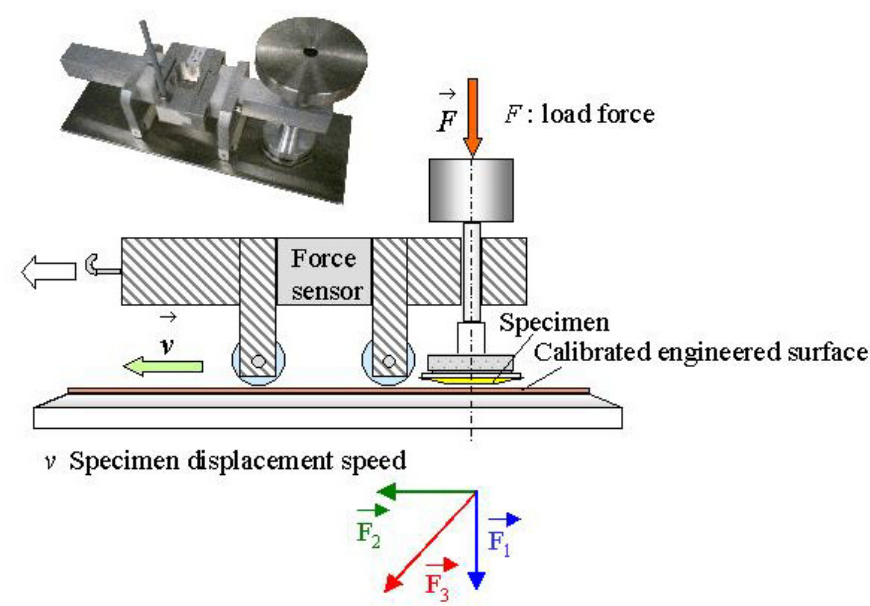

Figure 11: Sliding test: (side view) with schematic representation (bottom) of the force application, specimen displacement on calibrated engineered surface.

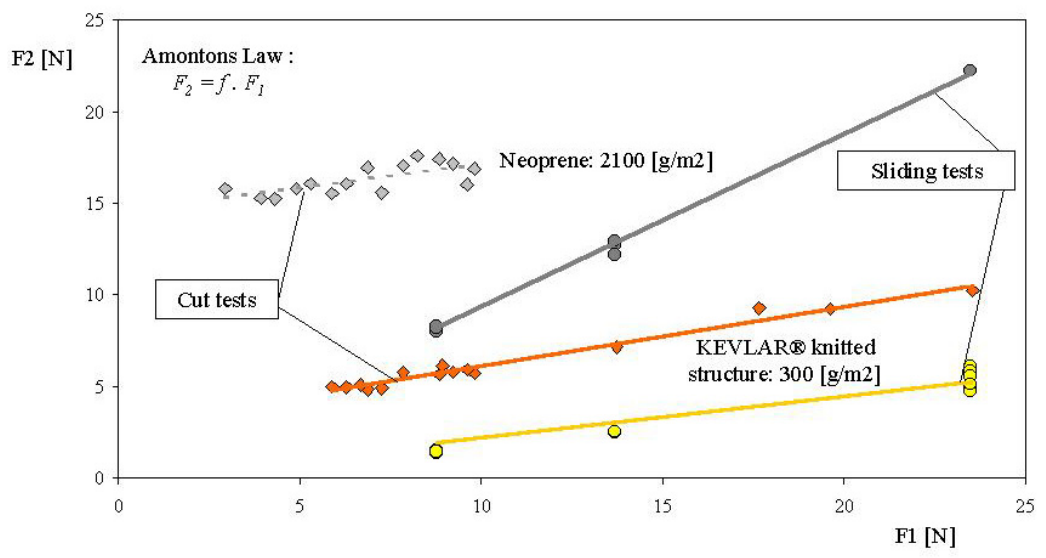

Figure 12: F2 / F1 ratio for various in case of cut testing and sliding tests.

Figure 12 outlines the similitude of the forces involved in the case of cutting versus the ones involved in the case of sliding. An amazing and revealing level of concordance can be observed, which means that one could definitely relates, to a certain extend, the surface wear and friction with the cut mechanism, which is by far more complex. This goes beyond the scope of this study, although 
during an accidental cut the sliding attributes of the personal protective equipment are determinant.

High molecular weight polyethylene textile structures are known to change their sliding and friction properties with moderate temperature as well as under significant abrasion and wear while other materials such as para-aramids tend to fibrillate bringing somehow and to a certain extend additional grip sometime to the detriment of appearance.

\section{Conclusion}

High performance materials based on high performance fibres significantly contribute to the emergence of new technologies under development today by fibre producers as well as converters and end-users relying on the unique attributes of para-aramids such as KEVLAR ${ }^{\circledR}$ in diversifying applications. Thermal and mechanical attributes reviewed, measured and partly discussed in the previous part of this work are contributing to better protection. Their "scientific contain" remains to be further explored.

Several tests methods, based on their equivalent standard procedures, were reviewed beyond their normal application frames.

In term of harmonization, the considered test conditions reinforce the appropriateness of the convergence towards a single norm, still allowing for multiple choices at the equipment and procedure levels to best represent the suitability of the material versus the expected cut protection level.

From this study one can appreciate as an improvement of the EN norm the possibility to test as per ASTM or ISO norm procedure especially when abrasive materials beyond level 3 are tested.

Similarly, the interchangeability of the ASTM and the ISO apparatus appears more and more useful as the fundamental understanding of test condition limits increases. Several situations studied in the present work support this aspect.

In terms of evolution, the emergence of high cut performance materials reinforced with glass or steel fibres, the market progression of coated protective equipments, the improvement of the test variability, and the substitution of the calibration material seems to be good focus points for reflection in the near term.

Several conclusions drawn in the course of this study may serve as a basis. The dulling effect of glass fibres towards the blade cutting edge can be misleading in terms of cut performance analysis. Similarly the artificial cut contribution of polymeric dots deposited on the textile substrate can also cause difficulties in terms of the cut performance interpretation. Those two specific situations are avoided when the blade cutting edge is in contact with the sample only once during the testing. ASTM or ISO are therefore more favourable in such cases.

A large amount of work remains to be done to fully integrate all the components of the identified improvements in a global understanding of how the cut mechanism and testing can evolve to be better predictive tools. Reference [16] provides more insight into this study. 
The present study has brought, hopefully, a refreshing scientific description of the state of the art knowledge of high performance fibres and related protective solutions with an effort to stimulate the desire to go beyond the knowledge and applications of today. There are properties of the aramids, a high performance family of fibres, which can still be optimised via the system approach. These materials have the potential to participate to the solutions of tomorrow.

\section{Acknowledgements}

The authors wish to express their gratitude to André Miret-Casas for his meticulous technical assistance and enthusiasm. Portia Yarborough, chairman of the F23 ASTM committee brought valuable comments in her review. DuPont fibre production centre in UK was instrumental to the independent evaluation and analysis of the materials tested.

Special thanks are also due to André Courgey from the European Automotive and Transportation Institute in France; his contribution to the wear and friction study is especially recognised.

\section{References}

[1] J. W. S. Hearle, High Performance Fibres, Woodhead Publishing Limited 2001, ISBN 1855735393.

[2] S. Rebouillat, M. Escoubes, R. Gauthier, J. Adhesion Sci. Technol., 10, 1996, pp. 635-650.

[3] S. Rebouillat, M. Escoubes, R. Gauthier, A. Vigier, Polymer, 36, 1995, pp. 4521-4523.

[4] S. Rebouillat, M. Escoubes, R. Gauthier, A. Vigier, J. App. Poly. Sci., 58, 1995, pp. 1305-1315.

[5] S. Rebouillat, J.B. Donnet, H. Guo, T.K. Wang, J. App. Poly. Sci., 67, 1998, pp. 487-500.

[6] S. Rebouillat, J.B. Donnet, T.K. Wang, Polymer, 38, 1997, pp. $2245-$ 2249.

[7] S. Rebouillat, J.C.M. Peng, J.B. Donnet, Polymer, 40, 1999, pp. 73417350 .

[8] American National Standard for Hand Protection Selection Criteria ANSI/ISEA 105-2000

[9] S.L. Kwolek, Am. Inst. Chem., The Chemist, 57(11), 1980, 9

[10] L. Penn, H.A. Newey, and T.T. Chiao, J. Mat. Sci, 11, 1976, 190.

[11] H.M. Caesar, Twaron Products BV, Chemical Fibre International, 50, 2000, 161-164

[12] S.L. Kwolek, P.W. Morgan, J.R. Schaefgen and L.W. Gulrich, Macromolecules, 10, 1977, 1390

[13] P.W. Morgan, Macromolecules, 10 (6), 1977, 1381.

[14] T.I. Bair, P.W. Morgan and F.I. Killian, Macromolecules, 10, 1977, 1396.

[15] S.L. Kwolek, US Patent Office, Pat No 3 671 542, 1972, Pat No 3819 587, 1974. 
[16] P. Yarborough, C. N. Nelson, Performance of Protective Clothing, Global Needs and Emerging Markets, ASTM International, STP1462, 2005.

[17] Tejani, N., Blocker, R, Schiffelbein, P., and Rivet, E., "Cut Protection Performance Test for Measuring Cut Resistance of Materials Used in Protective Clothing," Performance of Protective Clothing: Sixth Volume, ASTM STP 1273, Jeffrey O. Stull and Arthur D. Schwope, Eds., American Society for Testing and Materials, 1997.

[18] BS EN388, 1994, Protective gloves against mechanical risks

[19] ASTM F1790-97, Standard test method for measuring cut resistance of materials used in protective clothing

[20] ISO13997, ISO / TC 94/SC 13/WG 5 N 173, Protective clothingMechanical properties- Determination of resistance to cutting by sharp objects

[21] Lara, J., Turcot, D., Daigle, R, and Payot, F., "Comparison of Two Methods to Evaluate the Resistance of Protective Gloves to Cutting by Sharp Blades," Performance of Protective Clothing: Fifth Volume, ASTM STP 1237, James S. Johnson and S. Z. Mansdorf, Eds., American Society for Testing and Materials, 1996.

[22] Massé, S., Lara, J., Sirard, C., and Daigle, R., "Basic Principles Used in the Development of a New Cut-Test Apparatus for Standardization," Performance of Protective Clothing: Sixth Volume, ASTM STP 1273, Jeffrey O. Stull and Arthur D. Schwope, Eds., American Society for Testing and Materials, 1997. 